\title{
TRANSCRITICAL FLOW SIMULATION USING SHALLOW WATER EQUATION MODEL
}

\author{
Satria Damarnegara ${ }^{a^{*}}$, Rheszha Pahlavi Ali ${ }^{\mathrm{b}}$, Mohamad Bagus Ansori ${ }^{\mathrm{a}}$
}

\begin{abstract}
This paper shows the capabilities of DELFT3D-FLOW shallow water equation solver on transcritical flow. Two grid configurations are tested using a shock capturing numerical schemes that available on the solver. The simulation shows a good agreement with the analytical solution and proper grid resolution is needed to obtain a stable result. Implemented a shock capturing schemes is found to be critical to obtain a stable result. The model then used to simulate a real scale spillway chute channel of Logung Dam in Kudus-Central Java. The model could properly simulate the hydraulic jump, calculate the Froude number and stilling basin performance.
\end{abstract}

Keywords: Transcritical flow, DELFT3D, Shallow Water Equation

\section{INTRODUCTION}

Transcritical flow is one of popular test case for Shallow Water Equation (SWE) model because of its nature which consist of subcritical, critical and super critical flow. SWE allow efficient analysis of several flow problem in bigger scale. Trancscritical flow usually occur in flash flood event or in urban flood condition, where the flow is supercritical and return to subcritical because of influence of downstream condition. This transition described as a shock wave, where flow discontinuity occurred. Numerical SWE model is often used to solve hydraulic problem where transcritical flow usually occur. Hence, understanding the model capabilities to simulate transcritical flow is important, especially for hydraulic engineer.

Solving SWE numerically in transcritical flow problems is sensitive to flow discontinuities (e.g. hydraulic jump). Shock capturing method [1] introduced an artificial dissipation term to smear the discontinuity on standard Mac Cormack scheme. It tested on dam break flow problems and give a good result. However, numerical oscillation occurs near the front waves, which is reduced by selecting an appropriate artificial viscosity. Other method employed a Total Variation Diminishing (TVD) Mac Cormack scheme [2] and tested it on several case of transcritical flow. TVD Mac Cormack scheme obtained a good result on discontinuity compared with the Classical Mac Cormack Scheme. In recent times, many options are available on solving the flow discontinuity using a SWE model $[3,4,5,6]$.

DELFT3D-FLOW is a popular model made by Deltares [7] which already used worldwide and available as open source model. It solves the Non-Linear NavierStokes equation on curvilinear grid. When only one vertical layer is used, the model uses Shallow Water Equation where depth averaged velocity is used. The

aLaboratory of Water Resource and Coastal Engineering, Department of Civil Engineering, Institut Teknologi Sepuluh Nopember (ITS), ITS Campus, Sukolilo, Surabaya 60111, Indonesia.

Email: damarnegara@ce.its.ac.id

${ }^{\mathrm{b}}$ Graduate Program of Water Resources Engineering and Management, Department of Civil Engineering, Institut Teknologi Sepuluh Nopember (ITS), ITS Campus, Sukolilo, Surabaya 60111, Indonesia. model already applied in many project and validation case is included in the manual.

\section{RESEARCH SIGNIFICANCE}

In this paper, we investigate the applicability of DELFT3D-FLOW in transcritical flow problem. Two numerical schemes available are tested to show the importance of shock capturing method in transcritical flow simulation. Finally, the model is used to simulate a flow over spillway chute based on real case scenario.

\section{METHODOLOGY}

\section{A. MODEL DESCRIPTION}

DELFT3D-FLOW solves a non-linear shallow water equation with hydrostatic assumption for the pressure. The governing equation solves:

$$
\nabla \cdot \mathbf{u}=0
$$

The kinematic boundary condition for free surface and the bed are describe as:

$$
\begin{aligned}
\frac{\partial \zeta}{\partial t}+ & \frac{1}{\sqrt{G_{\xi \xi}} \sqrt{G_{\eta \eta}}} \frac{\partial\left((d+\zeta) U \sqrt{G_{\eta \eta}}\right)}{\partial \xi}+ \\
& \frac{1}{\sqrt{G_{\xi \xi}} \sqrt{G_{\eta \eta}}} \frac{\partial\left((d+\zeta) V \sqrt{G_{\xi \xi}}\right)}{\partial \eta}=(d+\zeta) Q
\end{aligned}
$$

With $U$ and $V$ are the depth averaged velocities for velocity vector $\mathbf{u}, d$ is the depth below reference level, $\zeta$ is the free surface elevation from reference level. $\sqrt{G_{\xi \xi}}$ and $\sqrt{G_{\eta \eta}}$ is correction term for curvilinear coordinate. Where $\xi$ and $\eta$ are the spatial direction of curvilinear system respectively. The momentum equation for $\xi$ and $\eta$ direction is:

$$
\begin{aligned}
& \frac{\partial u}{\partial t}+\frac{u}{\sqrt{G_{\xi \xi}}} \frac{\partial u}{\partial \xi}+\frac{v}{\sqrt{G_{\eta \eta}}} \frac{\partial u}{\partial \eta}-\frac{v^{2}}{\sqrt{G_{\xi \xi}} \sqrt{G_{\eta \eta}}} \frac{\partial \sqrt{G_{\eta \eta}}}{\partial \xi}+ \\
& \frac{u v}{\sqrt{G_{\xi \xi}} \sqrt{G_{\eta \eta}}} \frac{\partial \sqrt{G_{\xi \xi}}}{\partial \eta}-f v=-\frac{1}{\rho_{0} \sqrt{G_{\xi \xi}}} P_{\xi}+F_{\xi}+M_{\xi}
\end{aligned}
$$

and 
$\frac{\partial v}{\partial t}+\frac{u}{\sqrt{G_{\xi \xi}}} \frac{\partial v}{\partial \xi}+\frac{v}{\sqrt{G_{\eta \eta}}} \frac{\partial v}{\partial \eta}+\frac{u v}{\sqrt{G_{\xi \xi}} \sqrt{G_{\eta \eta}}} \frac{\partial \sqrt{G_{\eta \eta}}}{\partial \xi}+$

$\frac{v^{2}}{\sqrt{G_{\xi \xi}} \sqrt{G_{\eta \eta}}} \frac{\partial \sqrt{G_{\xi \xi}}}{\partial \eta}-f u=-\frac{1}{\rho_{0} \sqrt{G_{\eta \eta}}} P_{\eta}+F_{\eta}+M_{\eta}$

$P_{\xi, \eta}$ is the pressure gradient, $F_{\xi, \eta}$ is force due Reynold stress and $M_{\xi, \eta}$ is the external force sources.

Options of numerical schemes for solving the advection terms are available: WAQUA-scheme, Cyclic scheme and FLOOD scheme [8].

\section{B. SHOCK CAPTURING METHOD}

As mentioned previously, transcritical flow simulation includes a shock, where in hydraulic engineering problems mostly in form of hydraulic jump. FLOOD scheme uses a numerical approximation in order to obtain stable results in a rapidly varying flow. This method is second order accurate, however the accuracy will be reduced in extreme condition, where a slope limiter called Minmod is applied [8]. This limiter is important to prevent the oscillation that is well known in shock related problem.

The approximation of momentum conservation for normal advection term $u \frac{\partial u}{\partial \xi}$ based on a Control Volume $(\mathrm{CV})$ that is given by:

$$
\left.\frac{u}{\sqrt{G_{\xi \xi}}} \frac{\partial u}{\partial \xi}\right|_{m+\frac{1}{2}, n}=\frac{1}{H \sqrt{G_{\xi \xi}}} \frac{\partial q u}{\partial \xi}-\left.u \frac{\partial q}{\partial \xi}\right|_{m+\frac{1}{2}, n}
$$

In the discretization form:

$$
\begin{aligned}
& \left.\frac{u}{\sqrt{G_{\xi \xi}}} \frac{\partial u}{\partial \xi}\right|_{m+\frac{1}{2}, n}=\frac{2}{\left(H_{m, n}+H_{m+1, n}\right) \sqrt{G_{\xi \xi}}} \times \\
& \left\{\left(\frac{q_{m+1, n}^{-\xi} \widetilde{u}_{m+1, n}-q_{m, n}^{-\xi} \widetilde{u}_{m, n}}{\Delta \xi}\right)-u_{m+\frac{1}{2}, n}\left(\frac{q_{m+1, n}^{-\xi}-q_{m, n}^{-\xi}}{\Delta \xi}\right)\right\} \\
& q_{m, n}^{-\xi}=\frac{q_{m+\frac{1}{2}, n}+q_{m-\frac{1}{2}, n}}{2} \\
& \tilde{u}_{m, n}=\left\{\begin{array}{l}
u_{m-\frac{1}{2}, n}+\frac{1}{2} \psi\left(r_{u}\right)\left(u_{m-\frac{1}{2}, n}-u_{m-\frac{3}{2}, n}\right) \text { for } q_{m, n}^{-\xi} \geq 0 \\
u_{m+\frac{1}{2}, n}+\frac{1}{2} \psi\left(r_{u}\right)\left(u_{m+\frac{1}{2}, n}-u_{m+\frac{3}{2}, n}\right) \text { for } q_{m, n}^{-\xi}<0
\end{array}\right. \\
& \psi\left(r_{u}\right)=\max \left(0, \min \left(r_{u}, 1\right)\right)
\end{aligned}
$$

With $r_{u}$ is calculated as:

$$
r_{u}=\frac{u_{m+\frac{1}{2}, n}-u_{m-\frac{1}{2}, n}}{u_{m-\frac{1}{2}, n}-u_{m-\frac{3}{2}, n}}
$$

The limiter for negative flow direction is calculated as follows:

$$
\left.q\right|_{m+\frac{1}{2}, n}=\left.u\right|_{m+\frac{1}{2}, n} \frac{H_{m, n}+H_{m+1, n}}{2}
$$

Similarly, for cross advection term $v \frac{\partial u}{\partial \eta}$ the CV is given by:

$$
\left.\frac{v}{\sqrt{G_{\eta \eta}}} \frac{\partial u}{\partial \eta}\right|_{m+\frac{1}{2}, n}=\frac{1}{H \sqrt{G_{\eta \eta}}} \frac{\partial p u}{\partial \eta}-\left.u \frac{\partial p}{\partial \eta}\right|_{m+\frac{1}{2}, n}
$$

In the discretization form:

$$
\begin{gathered}
\left.\frac{v}{\sqrt{G_{\eta \eta}}} \frac{\partial u}{\partial \eta}\right|_{m+\frac{1}{2}, n}=\frac{2}{\left(H_{m, n}+H_{m+1, n}\right) \sqrt{G_{\eta \eta}}{ }_{m+\frac{1}{2}, n}} \times \\
\left\{\left(\frac{\left.p_{m+\frac{1}{2}, n+\frac{1}{2}{ }_{m+\frac{1}{2}, n+\frac{1}{2}}-q_{m+\frac{1}{2}, n-\frac{1}{2}}^{-\xi} m+\frac{1}{2}, n-\frac{1}{2}}^{\Delta \eta}\right)-}{\Delta \eta}\right)\right\} \\
\left.u_{m+\frac{1}{2}, n}\left(\frac{q_{m+\frac{1}{2}, n+\frac{1}{2}}^{-\xi} q_{m+\frac{1}{2}, n-\frac{1}{2}}^{-\xi}}{\Delta \eta}\right)\right\} \\
p_{m+\frac{1}{2}, n-\frac{1}{2}}^{-\xi} \frac{p_{m, n-\frac{1}{2}}+q_{m+1, n-\frac{1}{2}}}{2} \\
\tilde{u}_{m+\frac{1}{2}, n-\frac{1}{2}}=\left\{\begin{array}{l}
u_{m+\frac{1}{2}, n-1}+\frac{1}{2} \psi\left(r_{u}\right)\left(u_{m+\frac{1}{2}, n-1}-u_{m+\frac{1}{2}, n-2}\right) \text { for } p_{m+\frac{1}{2}, n-\frac{1}{2}}^{-\xi} \geq 0 \\
u_{m+\frac{1}{2}, n+1}+\frac{1}{2} \psi\left(r_{u}\right)\left(u_{m+\frac{1}{2}, n+1}-u_{m+\frac{1}{2}, n+2}\right) \text { for } p_{m+\frac{1}{2}, n-\frac{1}{2}}^{-\xi}<0 \\
\psi\left(r_{u}\right)=\max \left(0, \min \left(r_{u}, 1\right)\right)
\end{array}\right.
\end{gathered}
$$

With $r_{u}$ is calculated as:

$$
r_{u}=\frac{u_{m+\frac{1}{2}, n}-u_{m+\frac{1}{2}, n-1}}{u_{m+\frac{1}{2}, n-1}-u_{m+\frac{1}{2}, n-2}}
$$

The limiter for negative flow direction is calculated as follows:

$$
\left.p\right|_{m+\frac{1}{2}, n}=\left.v^{-\xi \eta}\right|_{m+\frac{1}{2}, n} \frac{H_{m, n}+H_{m+1, n}}{2}
$$

FLOOD scheme also introduced an energy head conservative discretization for similar CV () which is derived under steady state condition in 1D:

$$
\begin{aligned}
& \frac{q^{2}}{2 \sqrt{G_{\xi \xi}}}\left[\frac{1}{\left(H_{m+1, n}\right)^{2}}+\frac{1}{\left(H_{m, n}\right)^{2}}\right]_{\text {Energy Head Conservation }}= \\
& \lambda \frac{2 q^{2}}{\left(H_{m, n}+H_{m+1, n}\right) \sqrt{G_{\xi \xi}}}\left[\frac{1}{H_{m+1, n}}-\frac{1}{H_{m, n}}\right]_{\text {Momentum Conservation }}
\end{aligned}
$$

Detailed description of FLOOD advection scheme in DELFT3D is available in $[7,8]$

\section{ANALYSIS AND DISCUSSION}

\section{A. FLOW OVER A BUMP}

One popular case for shallow water equation is a flow over a bump [9]. It comprises of transition of supercritical flow into subcritical flow and hydraulic jump. The bump is defined as [10]:

$$
z(x)=z_{0}\left[\begin{array}{c}
\left.1-\frac{(x-5)^{2}}{4}\right] \\
0
\end{array} \begin{array}{c}
\text { for } 3 \mathrm{~m}<\mathrm{x}<8 \mathrm{~m} \\
\text { elsewhere }
\end{array}\right.
$$

Where $x$ is the horizontal direction of channel and $z_{0}=0.2$ $\mathrm{m}$ is the maximum elevation of the bump (Figure 1). For a transcritical flow with a shock the boundary condition is defined as follow: 
1. Discharge of $0.18 \mathrm{~m}^{3} / \mathrm{s}$ for the inlet boundary

2. Water level of $0.33 \mathrm{~m}$ for the outlet boundary

Test case parameter uses 2 grid variation. One case uses CYCLIC scheme in order to show the importance of FLOOD scheme on shock capturing. Complete model parameter is described in Table 1 . The analytical solution for frictionless and inviscid flow on flow over a bump is available [9]. In order to compare with the analytical solution, the simulation use very low manning number of 0.001 with no eddy viscosity. The initial condition of fluid is at rest with $U=0 \mathrm{~m} / \mathrm{s}$ and water level $=0.33$. The discharge then gradually increased linearly from $0 \mathrm{~m}^{3} / \mathrm{s}$ to $0.18 \mathrm{~m}^{3} / \mathrm{s}$ in $1 \mathrm{hr}$. The simulation reaches a steady state slightly after 1 hour (Figure 2).

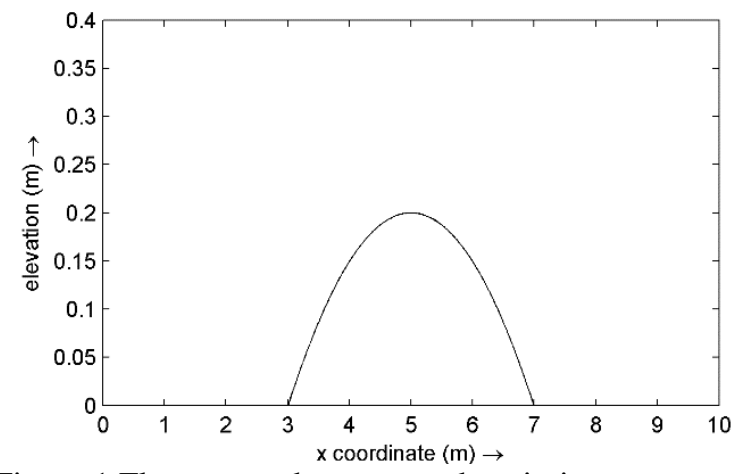

Figure 1 Flow over a bump case description

Table 1 Case list parameter for flow over bump case

\begin{tabular}{ccccc}
\hline Case No & $\Delta x(\mathrm{~m})$ & $\Delta y(\mathrm{~m})$ & Scheme & $\Delta \mathrm{t}(\mathrm{s})$ \\
\hline Case 01 & 0.10 & 0.10 & FLOOD & 0.006 \\
Case 02 & 0.03 & 0.03 & CYCLIC & 0.006 \\
Case 03 & 0.03 & 0.03 & FLOOD & 0.006 \\
\hline
\end{tabular}

Figure 3 shows the water surface elevation results compared with the analytical solution. The results show transcritical flow simulation should use a shock capturing scheme as Case 02 show an oscillating water surface elevation. Difference grid resolution also important. A grid which is too coarse will give erroneous results which is not a problem in finer grid as seen in Figure 4.

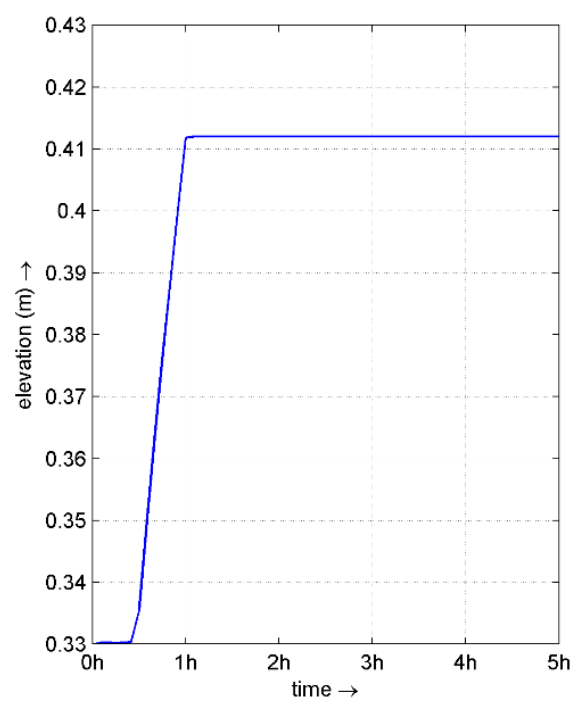

Figure 2 Water surface evolution at inlet for flow over a bump case

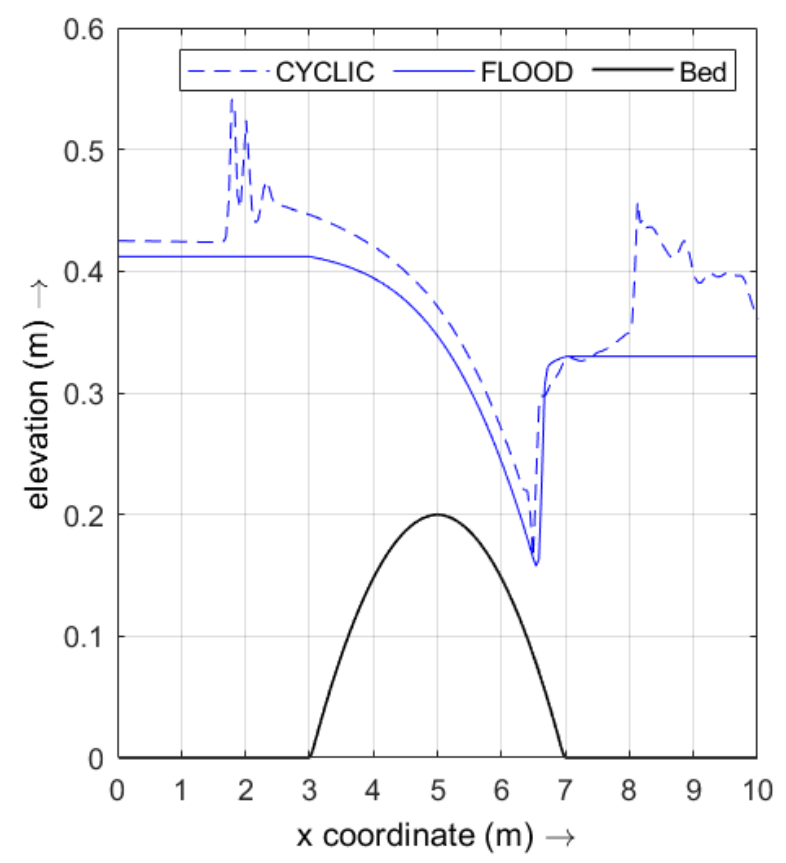

Figure 3 Water surface elevation comparison using CYCLIC and FLOOD schemes for flow over a bump case

Figure 5 shows the Froude number calculated from simulation. It shows the flow is transcritical where the Froude number is changed from subcritical before the bump, transition to supercritical on the bump and return to subcritical because of downstream boundary condition.

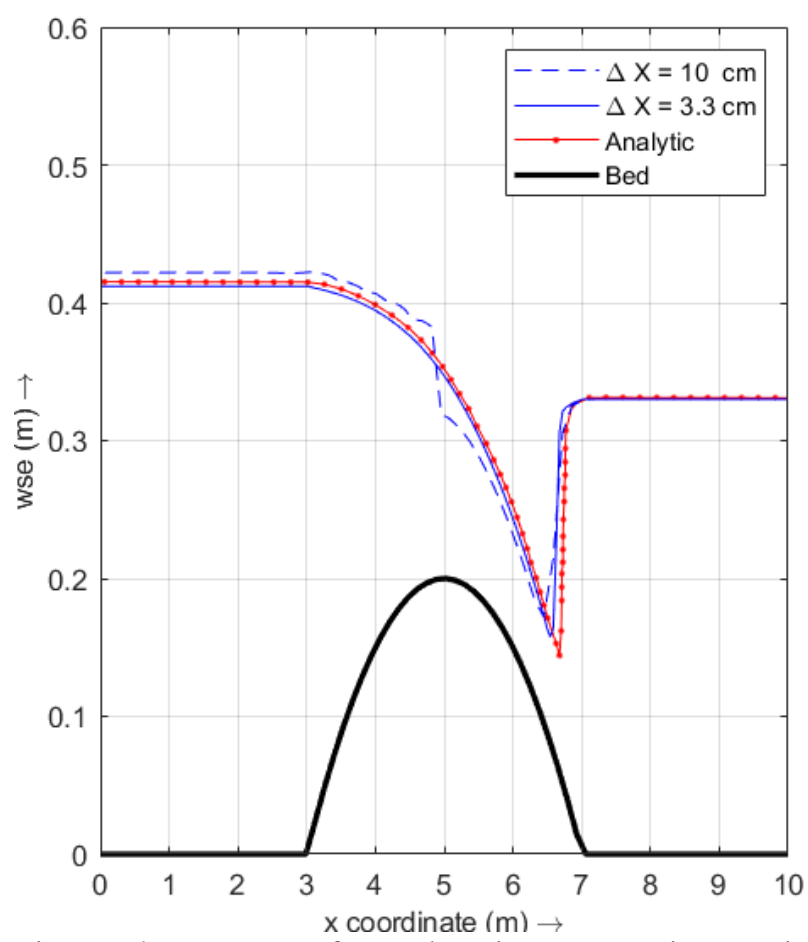

Figure 4 Water surface elevation comparison using different grid resolution for flow over a bump case 

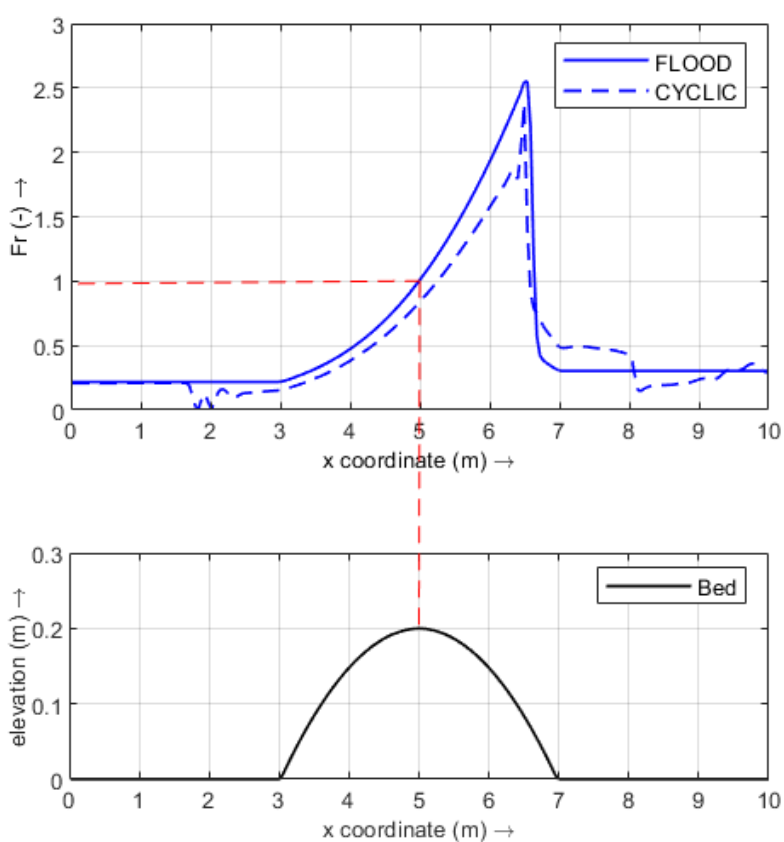

Figure 5 Froude number for flow over a bump case

\section{B. FLOW OVER SPILLWAY CHUTE}

DELFT3D model is applied to simulate a real scale of hypothetical spillway chute. The chute dimension is based on Logung Dam located in Kudus regency, Central Java. This simulation, however, did not represent the real design of the dam. The height difference of chute channel upstream to the downstream $\Delta H=43 \mathrm{~m}$ with 1:3 slope (Figure 6). The dam used side spillway which is not modeled in this simulation. The flow rate used in this simulation is $170.58 \mathrm{~m}^{3} / \mathrm{s}$ with designed tail water $=7.9 \mathrm{~m}$. The grid used in the simulation is $\Delta x=\Delta y=1 \mathrm{~m}$, with some refinement in $x$ direction of $0.5 \mathrm{~m}$ at the upstream and downstream of the chute (Figure 7).

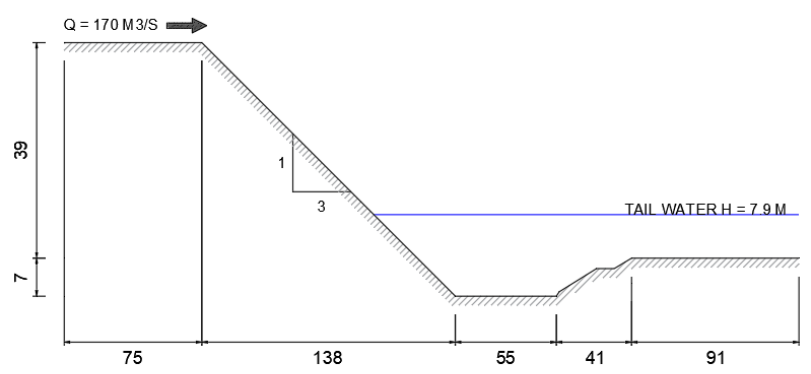

Figure 6 Case geometry for flow over spillway chute case

In order to obtain the steady state, an initial condition of water level of $44 \mathrm{~m}$ at rest is used. The water level is gradually reduced reaching the tail water height of $7.9 \mathrm{~m}$ for 18 hours. The simulation then continues for 48 hours. The flow rate boundary condition also increases gradually for 18 hours. Bottom friction is represented by Manning number of 0.013 for concrete material. Time step of 0.006 $\mathrm{s}$ is used to ensure CFL condition $<1$ is fulfilled. This is a disadvantage of DELFT3D which use explicit schemes for time integration.

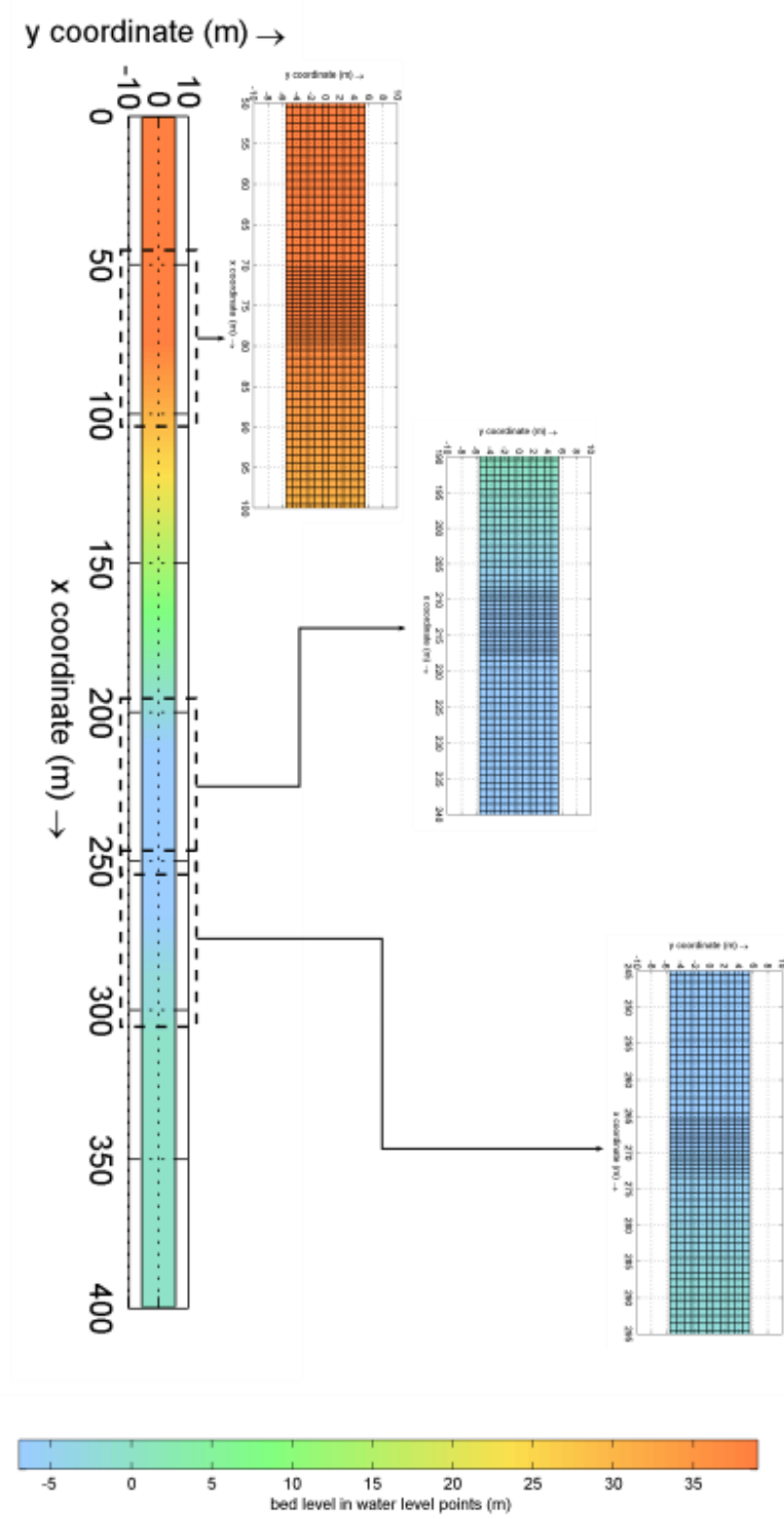

Figure 7 Grid configuration for flow over spillway chute case

The numerical simulation for 48 hours is done in 21 hours using Intel Core i $8^{\text {th }}$ Generation consisting of 6 physical cores and 12 threads. The simulation is done using distributed memory approach and it does not utilize full processor power. The steady state condition is reached after 18 hours of simulation time (Figure 8). The water surface elevation result shows the model could produce a realistic pattern of transcritical flow (Figure 9Figure 8). The models also could produce the hydraulic jump correctly. 


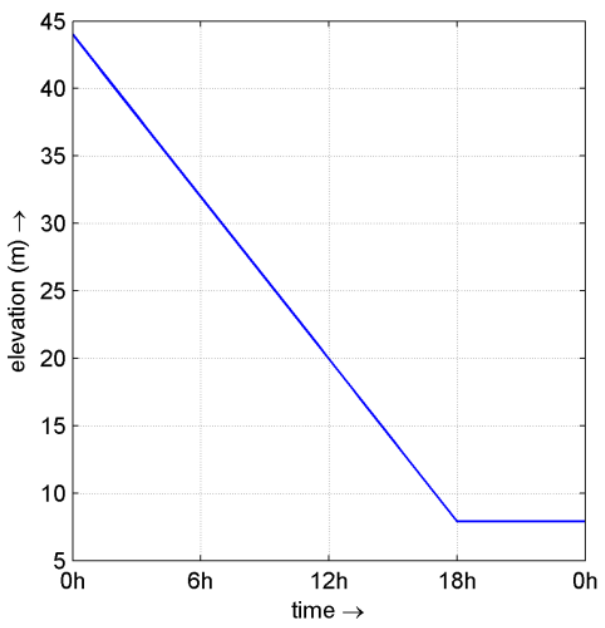

Figure 8 Water surface evolution for spillway chute case; Steady state condition is reach after 18 hours.
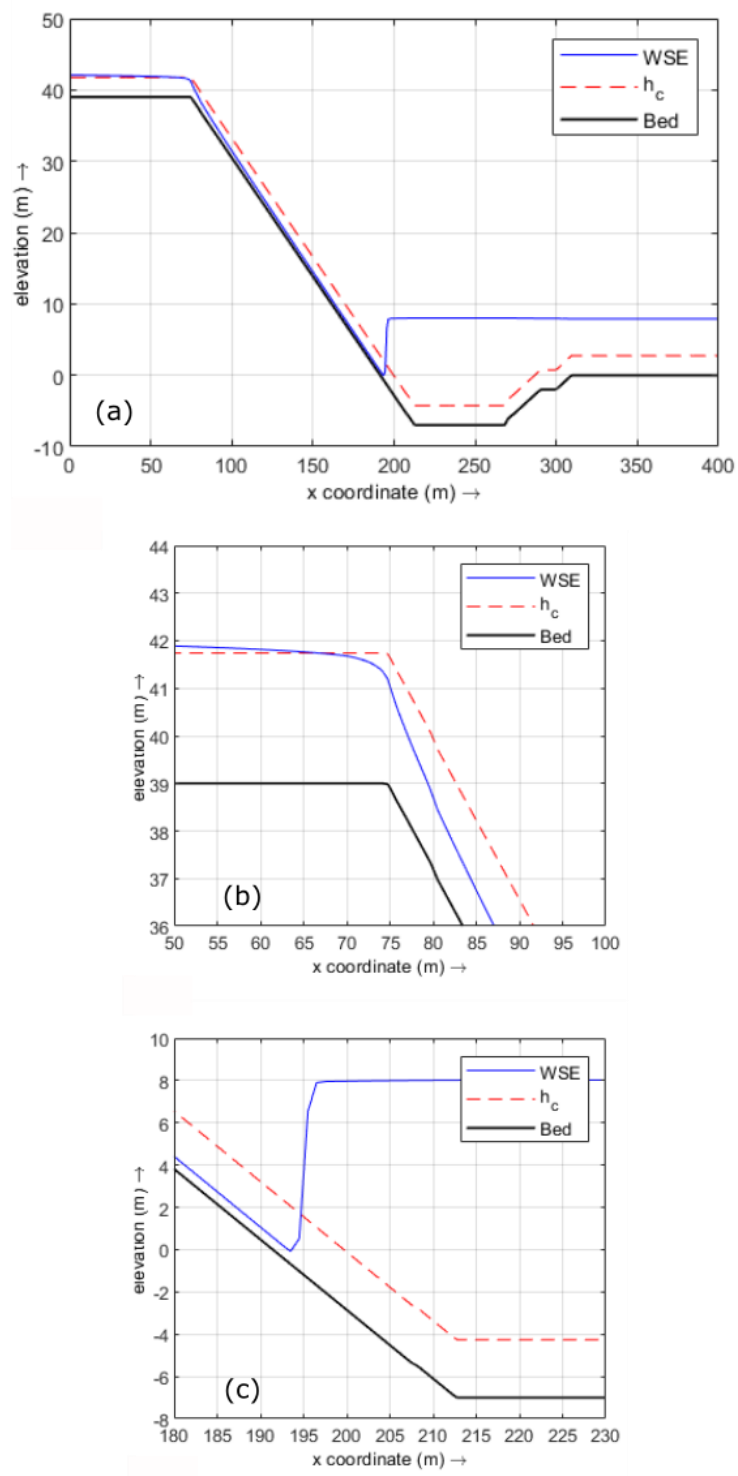

Figure 9 Water surface elevation for spillway chute case (solid line); critical depth is shown in dash line. (a) overall model (b) upstream of chute (c) hydraulic jump
The flow at chute is supercritical with maximum Froude number of 10 . The maximum flow velocity on the chute also obtained. The maximum velocity reach $24.5 \mathrm{~m} / \mathrm{s}$ at the chute downstream and reduce suddenly into $1.65 \mathrm{~m} / \mathrm{s}$ due to the hydraulic jump. Both the water surface and velocity show no oscillation on the solution, confirming the performance of FLOOD scheme shock capturing capabilities. The Froude number result also shows the stilling basin performance to dissipate energy. The Froude number near the outlet is subcritical with $\mathrm{Fr}=0.20$. This result should be validated further for example by using physical model. Indeed, it will be useful for preliminary identification of stilling basin design (Figure 10).

One important phenomenon in designing a hydraulic structure is the cavitation potential. Cavitation occur when the air is trapped and compressed induced a negative pressure near the structure. Because of hydrostatic assumption, cavitation potential is not directly available from the simulation results. However, designer could use the model results to have general description of cavitation potential.
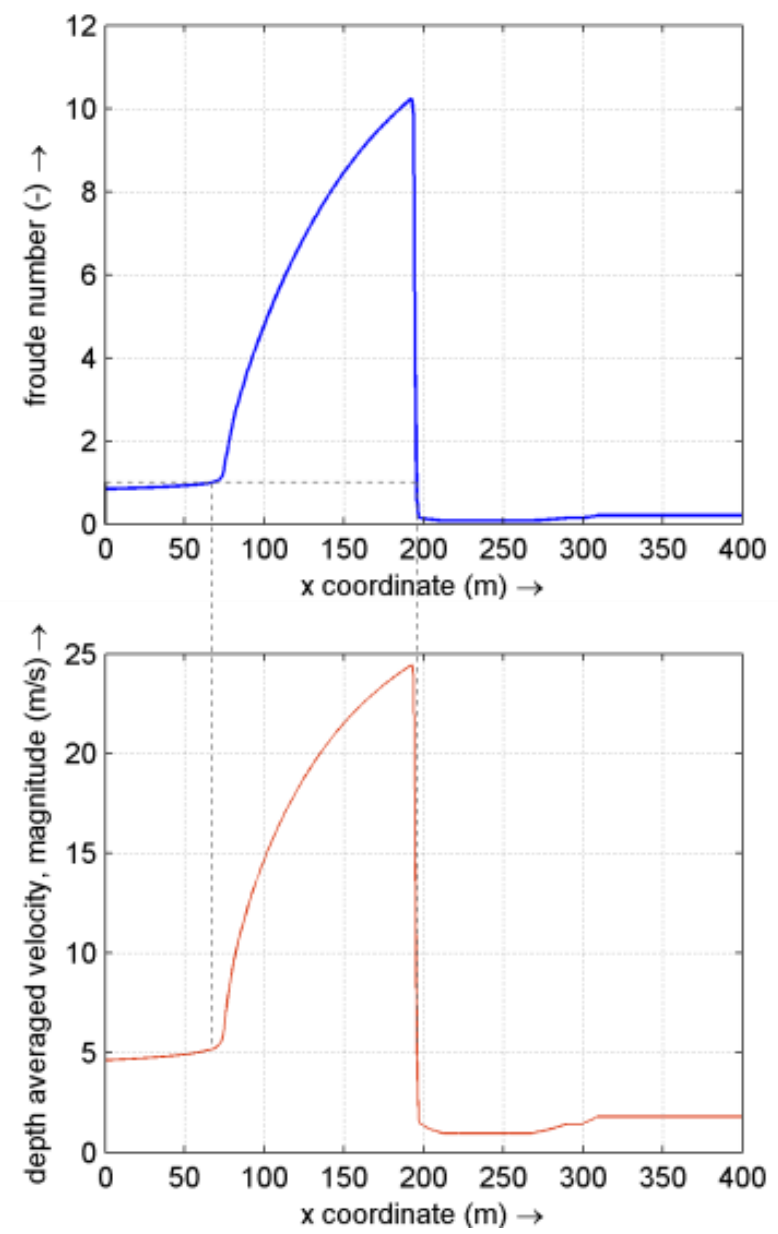

Figure 10 Froude number and flow velocity for spillway chute case; Note the Froude number and velocity change sharply at the hydraulic jump 


\section{CONCLUSION}

An open source Shallow Water Equation model DELFT3D is used to simulate a transcritical flow on open channel problem. The shock capturing capabilities of DELFT3D is implemented in FLOOD advection scheme. First test case is performed in frictionless and inviscid flow over a bump problem. The simulation shows that the simulation is not stable without FLOOD advection scheme implementation. Numerical oscillation is observed in the results with default CYCLIC scheme which is not the case for FLOOD scheme. Grid sensitivity results shows the model is not performing well in coarse grid hence a proper grid need to be considered. Another simulation for flow in spillway chute also performed. The simulation result in a good result. Water surface elevation results show the model could capture the hydraulic jump at chute downstream. The simulation also shows the model capabilities on simulating a high change of Froude number ( $\mathrm{Fr}=10$ and return into subcritical flow near the outlet). Hence, DELFT3D could be used to properly simulate a transcritical flow problem by implementing the shock capturing method available in the model.

\section{ACKNOWLEDGEMENT}

The author would like to acknowledge the support from Laboratory of Water Resource and Coastal Engineering Civil Engineering Department Institut Teknologi Sepuluh Nopember.

\section{REFERENCE}

[1] R. J. Fennema and M. H. Chaudhry, "Explicit methods for 2-D transient free surface flows," Journal of Hydraulic Engineering, vol. 116, no. 8, pp. 1013-1034, 1990.

[2] P. Garcia-Navarro, F. Alcrudo and J. M. Saviron, "1D open-channel flow simulation using TVDMcCormack scheme," Journal of Hydraulic Engineering, vol. 118, no. 10, pp. 1359-1372, 1992.

[3] E. A. Meselhe, F. Sotiropoulos and F. M. Holly Jr, "Numerical simulation of transcritical flow in open channels," Journal of hydraulic engineering, vol. 123, no. 9, pp. 774-783, 1997.

[4] D. A. Lyn and M. Altinakar, "St. Venant-Exner equations for near-critical and transcritical flows," Journal of Hydraulic Engineering, vol. 128, no. 6, pp. 579-587, 2002.

[5] Y. Xing and C.-W. Shu, "High order finite difference WENO schemes with the exact conservation property for the shallow water equations," 2005.

[6] D. Schwanenberg and M. Harms, "Discontinuous Galerkin Finite-Element Method for Transcritical Two-Dimensional Shallow Water Flows".

[7] Deltares, "Delft3D-FLOW Simulation of MultiDimensional Hydrodynamic Flows and Transport Phenomena Including Sediments, User Manual," Deltares Delft, The Netherlands, 2014.

[8] G. S. Stelling and S. P. Duinmeijer, "A staggered conservative scheme for every Froude number in rapidly varied shallow water flows," International
Journal for Numerical Methods in Fluids, vol. 43, no. 12, pp. 1329-1354, 30122003.

[9] O. Delestre, C. Lucas, P. Ksinant, F. Darboux, C. Laguerre, T. Vo, F. James and S. Cordier, "SWASHES: a compilation of shallow water analytic solutions for hydraulic and environmental studies," International Journal for Numerical Methods in Fluids, vol. 72, no. 3, pp. 269-300, 2013.

[10] R. Vacondio, B. D. Rogers, P. K. Stansby and P. Mignosa, "SPH Modeling of Shallow Flow with Open Boundaries for Practical Flood Simulation," 2012. 